\title{
Cambios cromáticos en la superficie cerámica
}

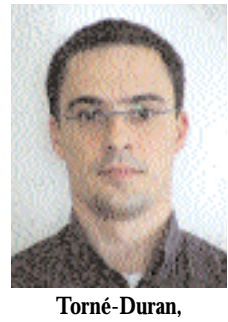

Sergi

\section{Chromatic changes on the ceramic surface}

\section{Torné- Duran, Sergi* \\ Escuin- Henar, Tomás** \\ Monreal-Nieto, J uliä*}

\author{
*Profesor Asociado. Facultad de Odon- \\ tología. Universidad de Barcelona. \\ **Profesor Titular. Facultad de Odon- \\ tología. Universidad de Barcelona.
}

\begin{abstract}
Resumen: El tallado insuficiente de un diente que va a recibir un retenedor en metal-porcelana supone confeccionar una restauración con adecuada morfología pero escaso grosor de porcelana o bien una restauración sobrecontorneada y grosor adecuado de porcelana. En este último caso, la consecución de una correcta oclusión puede obligar a un desgaste clínico intraoral de la porcelana lo que ocasiona una superficie altamente abrasiva que posteriormente requerirá un retoque por pulido mecánico o glaseado final. De Cualquier manera este retoque y pulido modifica la superficie de la porcelana y puede influenciar en el efecto cromático final de la restauración. Con esta base diseñamos una investigación para valorar los cambios de color ocasionados por el pulido de la porcelana. Las muestras fueron 95 especímenes de metal-porcelana combinando 2 colores, 6 aleaciones y 3 cerámicas diferentes. Todos se sometieron a un análisis colorimétrico. Los resultados muestran que existen diferencias de color entre aleaciones, cerámicas y tonos antes y después de un pulido de una superficie cerámica glaseada; siendo la aleación Cosmor la que más variación de color tiene y la aleación Wirón la que menos variación tiene. La cerámica que más ve influido su color por el pulido es la Vita VMK.
\end{abstract}

Palabras Clave: Cerámica, Color, Metal,Pulido.

Abstract: An insufficient tooth reduction for a subsequent PFM-retainer leads to the fabrication of either a restoration with an adequate morphology, but insufficient porcelain thickness or to an overcontoured restoration with an adequate porcelain thickness. In the last case, in order to achieve a proper occlusion it will be necessary to carry out an intraoral adjustment of the porcelain, producing a highly abrasive surface, which will need to be finished by mechanical polishing or final glazing. In both cases, these final adjustments and polishing steps will modify the porcelain surface and can have an impact on the final chromatic effect of the restoration. Based on this, we have designed a study to evaluate the colour changes associated to the polishing of the porcelain. It was carried out on 95 PFMsamples, combining two shades, six alloys and three ceramics. All were subjected to colorimetric analysis. The results show that there are differences in shade between the different alloys, ceramics and hues before and after the polishing of a glazed ceramic surface. The «Cosmor»-alloy shows the largest colour variation, while the «Wiron» alloy exhibits the smallest variation. The ceramic whose shade is more influenced by the polishing is «Vita VMK».

Key words: Ceramics, Colour, Polishing, Alloy.

BIBUD [1138-123X (2003)8:5; agosto-septiembre 469-592]

Torné-Duran S, Escuin-Henar T, Monreal-Nieto J. Cambios cromáticos en la superficie cerámica. RCOE 2003;8(5):487-496.
Sergi Torné Duran

C/ Aurora, 49 1-1

08700 Igualada. Barcelona 


\section{Introducción}

Ciertamente la introducción de materiales estéticos en Odontología ha supuesto uno de los avances más determinantes de cómo debemos ejercer y realizar las restauraciones dentarias. Estos materiales reúnen, junto a sus cualidades de manipulación, una exigencia, que por inespecífica u obvia, puede alterar nuestro deseo rehabilitador así como la satisfacción del paciente receptor de la prótesis.

Cuando un haz de luz encuentra la superficie de un objeto puede reflejarse, refractarse o transmitirse, o la combinación de las tres cosas. La luz proveniente del objeto incidirá en el ojo, se enfocará en la retina y se convertirá en impulsos nerviosos que se transmitirán al cerebro ${ }^{1}$.

Cuando consultan a un profesional para una restauración dental, los pacientes de hoy, buscan un resultado que sea biológicamente aceptable, funcionalmente perdurable y estéticamente agradable. Esto implica que el material restaurador sea de color dental. En esta línea la cerámica dental se ha situado como el material mas duradero y estético durante décadas².

Por otro lado, la calidad de cualquier porcelana depende de la elección de los componentes, del uso de las proporciones correctas de cada uno de ellos y del control del proceso de cocción.

Para lograr un acabado correcto de la cerámica y que nos ofrezca una superficie lisa y brillante, poco retentiva a depósitos orgánicos y de elevada estética se siguen tres técnicas que son el autoglaseado o glaseado intrínseco, el glaseado habitual o extrínseco y el pulido mecánico de la superficie ${ }^{3}$.

La técnica de uso en clínica que se ha propugnado para sustituir al glaseado es la del pulido de la restauración y consiste en el uso de una secuencia de gomas de pulir de grano progresivamente decreciente. Se considera la solución más idónea y necesaria en caso de la destrucción del glaseado de la porcelana por un ajuste postcementación ${ }^{3}$ aunque la erosión del glaseado también puede ocurrir en la boca.

En cualquier caso una superficie uniforme es esencial para llevar a su mínima expresión la retención de placa y la respuesta a los tejidos blandos. Así la pérdida de vidriado expone la superficie rugosa y a veces porosa del cuerpo de la porcelana. Las burbujas de aire existentes dentro de la porcelana son expuestas cuando se realiza el desgaste. Dependiendo del número y tamaño de las burbujas la superficie de la porcelana será más o menos irregular. Es por tanto mejor la porcelana cocida al vacío ya que disminuirá ostensiblemente el número de burbujas ${ }^{4}$.

Cuando se realiza el ajuste o desgaste de la cerámica, estamos realizando una abrasión de ésta. En un sentido estricto la palabra abrasión significa un desgaste por fricción de una superficie contra otra, y es lo que se realiza al retocar la cerámica intraoralmente. Algunos componentes de la cerámica tienen una dureza de 3 a 4 veces superior a la dureza del esmalte y pueden contribuir al desgaste de los dientes antagonistas naturales ${ }^{5}$.

La rugosidad de la superficie y la tensión superficial del material de la superficie $^{6}$ es importante para la adhesión de la placa bacteriana aunque el efecto no sea directo, ya que el efecto irritativo es de etiología bacteriana.

Un error frecuente es la inadecuada reducción del diente natural que impide la correcta estratificación de la cerámica y la colocación insuficiente de cerámica de forma que muchas veces nos obliga a un ajuste oclusal de la porcelana en clínica o intra-oralmente, de esta forma producimos una superficie altamente abrasiva y potencialmente destructiva sobre el esmalte antagonista.

Sin embargo, la norma de que toda superficie de porcelana ajustada debe de ser reglaseada para restaurar el acabado final no es siempre conveniente o posible 5* $^{*}$.

El ajuste de la porcelana en boca se realiza con diferentes instrumentos abrasivos, pero luego existe la necesidad de crear una superficie suave que nos reestablezca las ventajas de la porcelana6. La porcelana retocada intra-oralmente debería ser glaseada ya que se minimiza el potencial abrasivo contra los dientes naturales y reduce la irregularidad superficial, que pudiera causar la concentración de estres y fracturas ${ }^{7 *}$.

Monasky, en 1971, estudió la abrasión causada por la variación de combinación entre esmalte, cerámica y oro. Las conclusiones obtenidas fueron que la superficie de la cerámica más rugosa es la mas abrasiva, y a más pulido menos abrasiva. El oro se abrasiona más rápido que el esmalte si contacta con la cerámica ${ }^{8}$.

Einige, en 1979, postulaba que el glaseado fuera eliminado ya que la superficie del glaseado era mas dura que la cerámica inferior a este ${ }^{3}$. Pero estudios posteriores in vitro demostraron que no existe relación entre 
abrasión y dureza, por lo que la abrasión va determinada por la textura de la superficie.

El acabado final de las restauraciones, importante para la textura final, es determinante para dar naturalidad a la restauración. Este tratamiento final, que se manipula y altera de forma frecuente debido al ajuste de la oclusión y al sobre-contorneado de la prótesis, que se retoca, se pule y se le da brillo en clínica, parece que debería influir de alguna manera en el resultado cromático final en mayor $o$ menor medida.

Por lo tanto, ante la importancia y necesidad que este aspecto descriptivo tiene en la comunicación entre la clínica odontológica y el laboratorio protésico, nos planteamos una hipótesis de trabajo que nos permita, en la medida de lo posible, discernir las variaciones del color de la cerámica dental cuando ésta sea pulida o glaseada, durante el acabado final. La hipótesis será la siguiente:

- El tratamiento final que se realice a la restauración no influye en el color final de la misma.

Ante la hipótesis de trabajo citada, nuestro objetivo será la comprobación de la misma, mediante el diseño de un método que nos permita evaluar los valores espectrales de reflectancia de los distintos especimenes. Dichos especimenes serán sometidos a variaciones controladas, tanto de la aleación metálica utilizada en su elaboración, tipo de cerámica, grosor, color de la cerámica, acabado final y luz incidente.

Los resultados obtenidos serán tratados mediante pruebas de inferencia estadística que nos permitan dilucidar las posibles relaciones entre la utilización de distintas aleaciones,

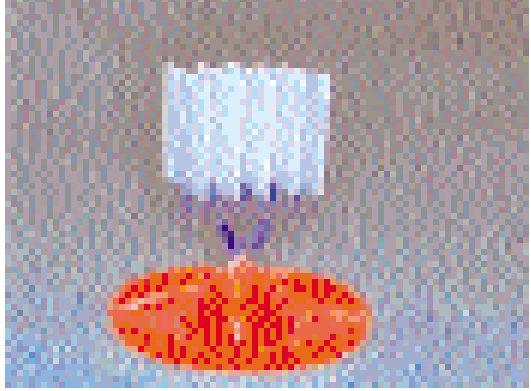

Figura 1.Muestras de plástico calcinable sin residuos para ser colocadas en el cilindro

cerámicas, colores, luces y valorar de una manera global si el resultado final del color de la cerámica es influenciado según el tratamiento final a que sea expuesto.

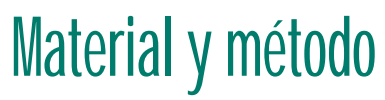

Se prepararon 95 muestras de plástico calcinable, de acetato sin residuos, de $20 \times 20 \times 0,5 \mathrm{~mm}$. Se colocaron cinco muestras en cada cilindro, para posteriormente vaciarlas en revestimiento ligado a base de fosfatos, sin grafito, (Deguvest HF, Degussa) (fig. 1) y colocar dichos cilindros en el horno de precalentamiento, según la recomendación del fabricante, las cuales serían coladas en las diferentes aleaciones metálicas objeto de este estudio.

Las aleaciones metálicas estudiadas, se escogieron en función de su composición porcentual, entre aquellas que son utilizadas más frecuentemente para la realización de restauraciones metalo-cerámicas:

- Aleación de alto contenido en oro (78,5\%) (Esteticor Cosmor $\mathrm{H}$, Cendres et Metaux S.A. Biel-Bienne, Suiza).
- Aleación de alto contenido en paladio, sin plata (77,8\%) (Esteticor Biennor, Cendres et Metaux S.A. Biel-Bienne, Suiza).

- Aleación de alto contenido en paladio, con plata $(53,6 \%$ de $\mathrm{Pd} ; 37,6$ de Ag) (Esteticor Actual, Cendres et Metaux S.A. Biel-Bienne, Suiza).

- Aleación de cromo-niquel, (Cr 1214\% Ni 74-78\% Be 1,8\%) (Rexillium III, Jeneric Gold Co. P.o.Box,724, Wallingford, Conn. 06492, EE.UU).

- Aleación de cromo-niquel, $(\mathrm{Cr}$ $12,5 \% \mathrm{Ni} 73,5 \%$ Be 1,7\%) (Piscis, Ivoclar-Vivodent, Schaan, Liechtenstein).

- Aleación de cromo-niquel, $(\mathrm{Cr}$ 22,5\% Ni 65,5\% Mo 9,5\%) (Wiron 99, BEGO, Bremer, Germany).

Con estas aleaciones, siguiendo las indicaciones de tratamiento térmico indicadas por el fabricante de cada una de las mismas, se confeccionaron un total de 95 placas de $2 \mathrm{~cm} \times 2 \mathrm{~cm}$ $x$ 0,5 $\mathrm{mm}$, que fueron procesadas, 0 coladas por inducción de su fusión.

Antes de realizar la oxidación de cada una de las muestras, se repasaron las superficies metálicas con instrumentos rotatorios diamantados, y piedras de carburo de tungsteno, hasta conseguir una superficie homogénea.

Una vez repasado el metal se realizó la oxidación de las diferentes placas, con un horno P-90 de Ivoclar (Ivoclar-Vivodent, Schaan, Liechtenstein), variando los programas de oxidación para las diferentes aleaciones, según las indicaciones del fabricante para cada una de ellas.

Una vez oxidado el metal se aplicó una primera capa de opaquer en pasta, de color B1 y A3 muy fluido con un pincel sobre cada una de las placas.

Se usaron dos tonos de color cerá- 


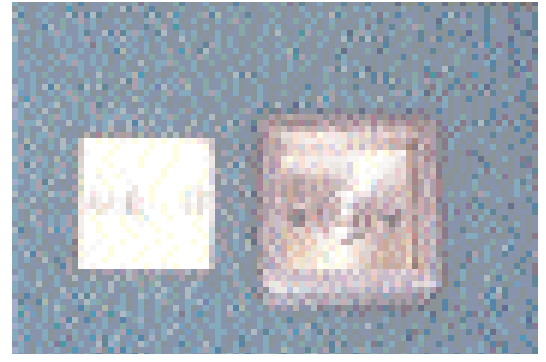

Figura 2. Aspecto del molde metálico con el tornillo calibrado.

mico, el color A3 y el color B1. El criterio seguido es usar el tono más claro, (B1) y el tono de uso más frecuente $(\mathrm{A} 3)^{9,10}$.

Las cerámicas utilizadas, cuya composición química facilitada por las casas comerciales se describe al lado de cada una de ellas, fueron las siguientes:

- IVOCLAR: IPS classic

- VITA VMK95

- VITA OMEGA 900

Se procedió a la primera cocción del opaquer, y posteriormente se aplicó una segunda capa de opaquer, pero esta vez con una espátula de vidrio para evitar la inclusión de burbujas de aire y se procedió a su cocción según temperaturas recomendadas por el fabricante.

Para la aplicación de la cerámica de cuerpo se fabricó un instrumento o cubeta de acero inoxidable, que sirvió de molde para la aplicación de la cerámica de cuerpo y con el cual pudimos controlar el grosor de la capa de cerámica que quisimos aplicar.

El molde metálico tenía un aditamento interno para regular la altura de relleno del mismo a través de un tornillo calibrado que nos permite aumentar o disminuir su profundidad a razón de $1 \mathrm{~mm}$ por vuelta completa de la rosca del tornillo (fig. 2).

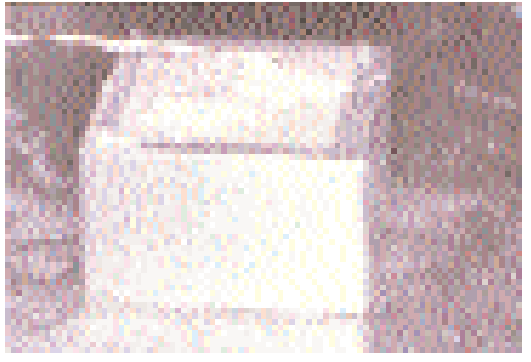

Figura 3: Cerámica de cuerpo sobre la plancha metálica en el interior del molde.

Se colocó cada plancha metálica en el interior del molde, calibrado previamente para conseguir un grosor de $1,68 \mathrm{~mm}$ y $2,80 \mathrm{~mm}$ (fig. 3 ), y se rellenó de cerámica de cuerpo con la ayuda de una espátula. Cada plancha se condensó mediante vibración con ayuda del mango de una espátula de Le Cron, hasta conseguir que no apareciera humedad en la superficie. Se secaron con un papel absorbente y se eliminó el exceso de dentina, controlando así el grosor total de los especímenes.

Se colocaron los discos en una platina y se realizó la cocción en un horno P-90 calibrado previamente realizando el glaseado según los programas de cocción recomendados por el fabricante.

Todos los especímenes fueron realizados por el mismo operador, en el Laboratorio de Prótesis de la Facultad de Odontología de la Universidad de Barcelona.

Una vez obtenidas las placas, medimos el grosor total de cada una de ellas en tres puntos diferentes escogidos al azar con un calibrador de 0,01 mm. (fig. 4 ).

Para poder analizar el color de cada una de las muestras se hizo la lectura de las mismas con un espectrofotómetro SHIMADZU UV-2101 (Tokio, Japón) de los servicios Científi-

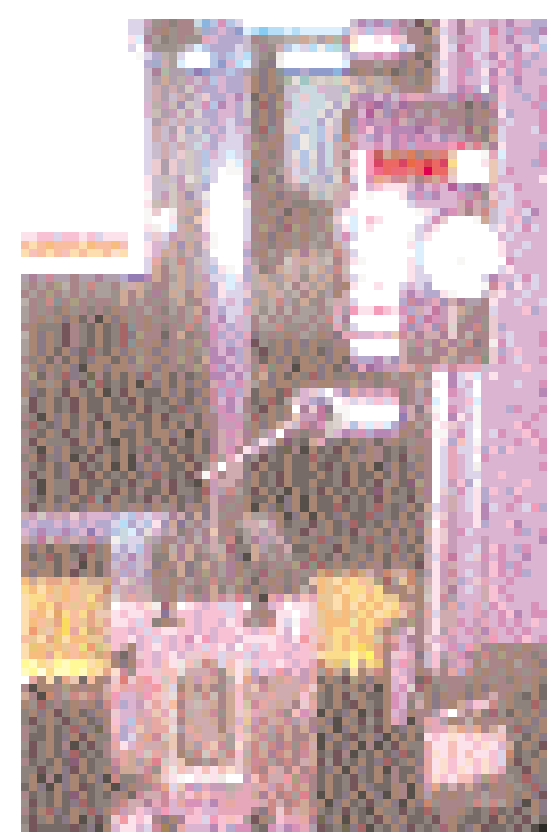

Figura 4. Aspecto del calibrador.

co Técnicos de la Universidad de Barcelona.

Se realizaron dos lecturas de cada placa, de la luz reflejada en la zona del espectro visible (380 nm a $700 \mathrm{~nm}$ ), a intervalos de $10 \mathrm{~nm}$ (curva de reflectancia).

Para el análisis de la fiabilidad del espectrofotómetro se realizaron 10 lecturas de una misma muestra, más 10 lecturas de una misma muestra en diferente posición y finalmente cinco lecturas en momentos diferentes.

Una vez obtenidas las curvas de reflectancia (dos lecturas de cada placa), se procedió a su transformación matemática al sistema CIE-LAB.

Una vez obtenidos los valores $L^{*}$, $a^{*}, b^{*}$, de cada especímen ( $L^{*}$ es una variable lumínica proporcional al brillo o luminosidad del sistema Munsell; a* y b* son coordenadas de cromaticidad aunque no se relacionan con los del sistema Munsell. La coordenada a* corresponde al eje rojo-verde, la coor- 


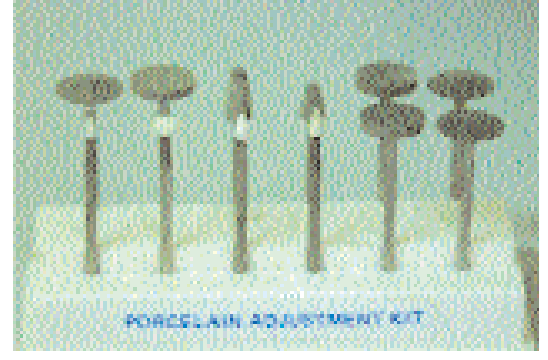

Figura 5. Surtido de fresas para hacer el pulido cerámico.

denada b* corresponde al eje amarillo-azul). Se realizó una tabla resumen de todas lecturas en unidades CIE$L A B$, a partir de la cual obtuvimos la matriz de resultados que será analizada estadísticamente con el programa SPSS V.10 para windows.

Una vez obtenidos y analizados los resultados y valorando el color resultante de todos los especimenes con un acabado final glaseado, procedemos a una fase que se basó en el pulido de los 95 especímenes anteriores.

El pulido consistió en un proceso de grabado con una fresa de diamante con una turbina durante 60 segundos cada muestra. Este grabado se realizó de derecha a izquierda, de izquierda a derecha, de arriba abajo y de abajo a arriba repartiendo el tiempo de forma igual en cada sentido.

Se realizó un pulido de la superficie de cada muestra mediante un surtido de fresas de pulido para cerámica (SHOFU), ya usado en estudios anteriores y demostrada su validez ${ }^{11}$ (fig. 5).

El proceso de pulido se hizo con cuatro fresas de silicona de distinto grado de abrasión, comenzando en el orden indicado por el fabricante, durante un periodo de 90 segundos cada fresa, repartido entre los cuatro sentidos posibles. El pulido se realizó en los cuatro sentidos con el mismo tiempo en cada sentido.
Tras el pulido se realizó la lectura colorimétrica de las muestras de igual forma que en los anteriores estudios obteniendo los resultados que indicaremos en el apartado correspondiente.

Para tener una visión gráfica del proceso de pulido realizado se observaron las muestras con un microscopio electrónico de barrido. Las muestras fueron preparadas para su observación con polvo de oro por el personal de los servicios científico técnicos de la Universidad de Barcelona.

\section{Análisis estadístico}

Los datos han sido analizados en la Unidad de Bioestadística de la Facultad de Medicina en el Hospital Clínico de Barcelona.

En primer lugar se evaluó la fiabilidad de la máquina midiendo una misma muestra varias veces (varianza intra medida), una misma muestra en posiciones diferentes para ver la influencia tanto de la superficie, como de la zona leída (varianza entre medida) y, por último si variaron los resultados al leer una misma muestra en momentos diferentes. La dispersión obtenida nos permite considerar una distribución de los datos adecuada para seguir con nuestro estudio estadístico.

Dado que de cada muestra estudiada se disponen de dos medidas para cada componente de definición del color, se ha calculado $L^{*}, a^{*}$ y b* como la media aritmética de las dos observaciones, realizando tablas descriptivas de los resultados obtenidos en unidades CIE-Lab .

Con estos nuevos valores se ha calculado la variable diferencia de color o DE* que resume las diferencias de color entre el tipo de luz día o
D65 y las otras dos luces (A y C). Estas luces son: una luz tipo día de 6504으 que coge la zona del espectro de los ultravioletas (D65), una luz día de 6774 K y sin el espectro ultravioleta (C) y una luz de $2856^{\circ} \mathrm{K}$ de tipo tungsteno (A). De manera que obtenemos una ma-triz de resultados que es la que analizaremos estadísticamente.

El estudio, se ha dividido en dos bloques. En el primero se estudiaron los metales condicionados a que el diente contenga las características siguientes: grosor 1.68, color B1 y cerámica Ivoclar. Las variables se han descrito con la media y la desviación típica. Se ha estudiado la diferencia entre el antes y el después de cada aleación con la $t$ de Student y la diferencia entre aleaciones mediante un análisis de la varianza. Las comparaciones múltiples se han realizado usando la corrección de Tuckey.

En el segundo se estudiaron los metales y las cerámicas condicionado a que el diente contenga las siguientes características: grosor 2.8 y color A3. Las variables se han descrito, también, con la media y la desviación típica. Se ha estudiado la diferencia entre el antes y el después de las cerámicas en función de los metales con la t de Student y la diferencia entre cerámicas en función, también, de las aleaciones con un análisis de la varianza. Las comparaciones múltiples se han realizado usando la corrección de Tuckey. Se ha complementado el estudio con gráficos de medias e intervalos de confianza.

Para la creación de la base de datos se ha utilizado el programa Excel y para el análisis estadístico el programa SPSS versión 9.0. Se ha tomado un nivel de significación del 0.05 $(\alpha=0.05)$. 


\section{Resultados}

Tras la explicación de los materiales y métodos estadísticos utilizados, se obtuvieron los siguientes resultados, que serán descritos de forma consecutiva según su realización y obtención.

El grosor medio de las muestras en el primer bloque (placa aleación-cerámica) fue de $1,68 \pm 0,23 \mathrm{~mm}$, en el segundo bloque fue de 2,8 $\pm 0,23 \mathrm{~mm}$.

Identificamos la aleación y el color de la siguiente forma:

$r \quad$ Rexillium III

a Actual

b Esteticor Biennor

c Cosmor $\mathrm{H}$

w Piscis Williams

wi Wirón

iv Cerámica ivoclar

v Cerámica vita VMK95

vo Cerámica omega 900

b1 Color B1

a3 Color A3

En primer lugar se describirán los resultados de las muestras de grosor 1,68 mm, color B1 y A3 y cerámica Ivoclar para cada una de las luces $C$ y $A$.

En segundo lugar se procesaron las muestras de grosor $2,8 \mathrm{~mm}$, color $\mathrm{A} 3$ y cerámica diferentes para cada una de las luces $\mathrm{C}$ y $\mathrm{A}$.

\section{1) GROSOR: 1,68 COLOR: B1 CERÁ-MICA: IVOCLAR}

\section{1) Luz C-METALES}

Se observó que en la diferencia de color en luz $C$ de los metales variaba significativamente entre el antes y el después del pulido.

1.1.1) Comparación del efecto pulido entre metales:

Hay diferencias entre «Actual»y «Cosmor», esto quiere decir que la pér-

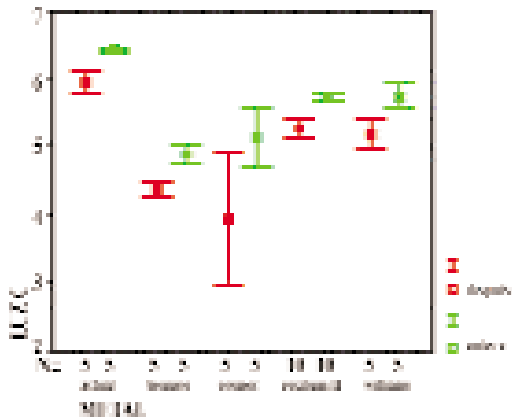

Figura 6.

dida de diferencia de color del «Cosmor» es mayor que la del «Actual». L mismo ocurre entre los metales «Biennor» versus «Cosmor», «Rexilium» verSus «Cosmor» y «Williams» versus «Cosmor», donde la pérdida del «cosmo» es superior a los demás.

1.1.2) Gráfico medias e intervalos de confianza de los metales (antes y después) (fig. 6).

Se puede concluir pues que el «cosmor» es diferente a todos los demás y que su pérdida de color es mayor a las otras.

\section{2) Luz A-METALES}

1.2.1) Análisis del efecto pulido para cada metal:

Todos los metales presentan diferencias significativas de color entre el antes y el después observadas en luz $A$. «Cosmor» es el que presenta diferencias mayores.

1.2.2) Comparación del efecto pulido entre metales:

Hay diferencias significativas entre los metales.

Existen diferencias entre «cosmor» $\mathrm{y}$ «Bienor» $\mathrm{y}$ 《Cosmor»y «Rexilium III», siendo la pérdida de diferéncia de color en luz A del «Cosmor» superior a la de los otros dos metales.

1.2.3) Gráfico medias e intervalos de confianza de los metales (antes y después) (fig. 7).

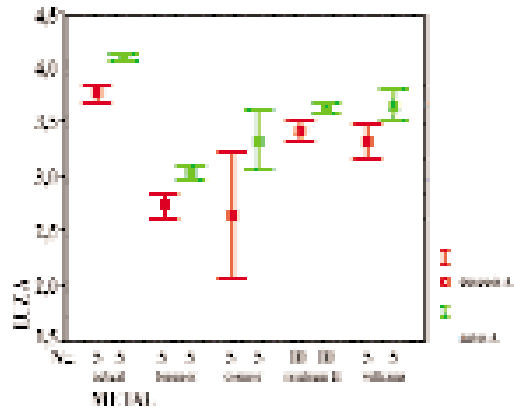

Figura 7.

Se concluye que «Cosmor» es el que presenta mayor variación de color al pulir la cerámica .

\section{2) GROSOR: 2,8 COLOR: A3}

\section{1) Luz C-METALES Y CERÁMICAS}

Se sabe que existe interacción entre metal y cerámica, eso implica analizar todas las situaciones experimentales.

2.1.1) Descriptiva de cada situación experimental antes y después:

Análisis del efecto pulido para cada situación experimental: se observó que respecto al «Biennor» la única cerámica con diferencias es la «Wmk». Respecto al metal «Piscis», «lvoclar»y «Wmk» presentaron diferencias. Finalmente «Wiron» no tuvo ninguna diferencia.

2.1.3) Comparación del efecto pulido entre situaciones experimentales que presentan diferencias entre antes y después:

No hay diferencias significativas entre las situaciones experimentales que presentaban diferencias entre antes y después.

2.1.4) Gráficos de medias e intervalos de confianza:

2.1.4.1) Metal «Biennor» (antes y después) (fig. 8)

2.1.4.2) Metal «Piscis« (antes y después) (fig. 9) 


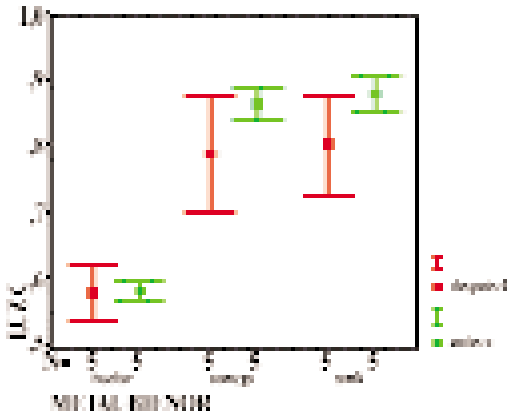

Figura 8.

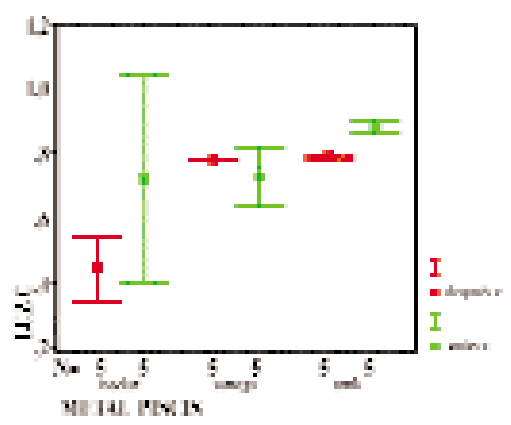

Figura 9.

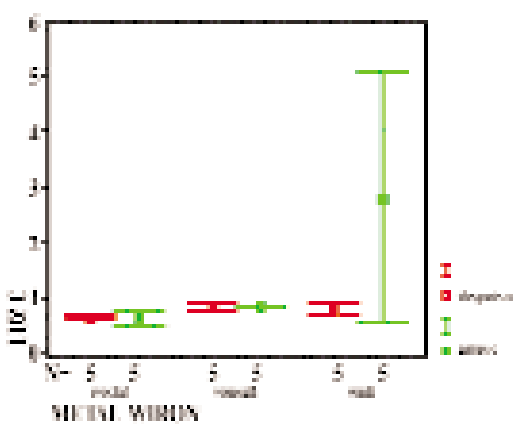

Figura 10.

2.1.4.3) Metal «Wiron« (antes y después) (fig. 10).

\section{2) LUz A-METALES Y CERÁMICAS}

Se sabe que hay interacción entre metal y cerámica, eso implica analizar todas las situaciones experimentales.

2.2.1) Análisis del efecto pulido para cada situación experimental:

Con el metal «Biennor « hay diferencias entre el antes y el después en

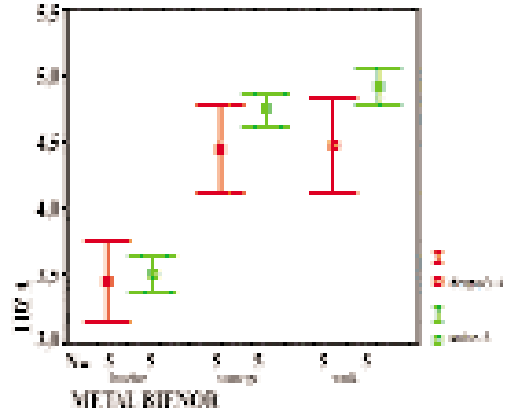

Figura 11.

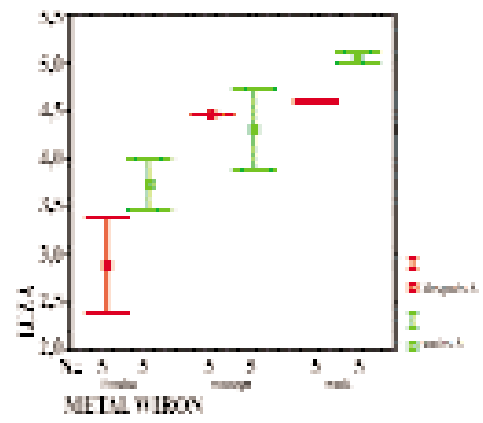

Figura 12.

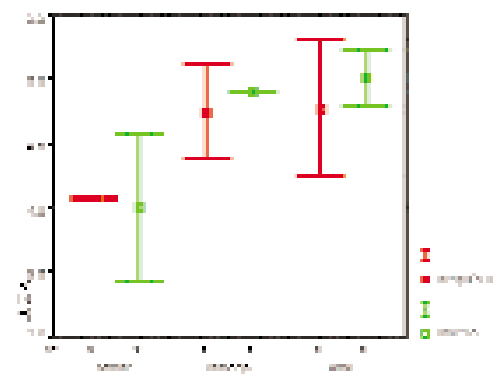

Figura 13.

la cerámica «Wmk». Con «Piscis» hay diferencias en las cerámicas «voclar» $\mathrm{y} \ll W m k »$. Finalmente con «Wiron» ninguna cerámica presenta diferencias.

2.2.2) Comparación del efecto pulido entre situaciones experimentales que presentan diferencias entre antes y después:

No hay diferencias significativas entre las situaciones experimentales que presentan diferencias entre antes y después.

2.2.3) Gráficos de medias e intervalos de confianza:

2.2.3.1) Metal «Bienor» (antes y después) (fig. 11).

2.2.3.2) Metal «Piscis» (antes y después) (fig. 12).

2.2.3.3) Metal «Wiron» (antes y después) (fig. 13)

\section{Resultados de microscopia electrónica de barrido}

Tras analizar estadísticamente los resultados se ha creído conveniente realizar observaciones bajo microscopia electrónica de barrido para tener una imagen real y ampliada de las superficies cerámicas pulidas y glaseadas, para poder valorar el cambio externo que supone (figs. 14 y 15)

\section{Discusión}

La predictibilidad de los resultados estéticos, se ha considerado en Odontología como uno de los factores de calidad de las restauraciones protésicas.

Por ello, los tonos escogidos por el odontólogo, con ayuda de los muestrarios de matices o «juegos de colores», no nos acercan a la realidad del color, dando lugar a la necesidad de realizar guías individualizadas para comparar nuestros modelos a restaurar, no con guías estándares, sino con la cerámica y el grosor que utilizamos habitualmente; y controlar así la mayoría de factores influyentes ${ }^{12}$.

El ojo humano no es adecuado para una determinación objetiva de los colores $^{13}$. Al Dr Bruce Clark le corresponde el haber sido el primero en someter dientes naturales a medi- 
ciones colorimétricas. No obstante, es muy difícil conseguir la igualación perfecta del color, ya sea por que las cerámicas dentales no abarcan el mismo área de color que los dientes naturales ${ }^{14}$, por la existencia de una visión anómala del color por parte del profesional, o bien por la iluminación en que la lectura del color se realiza.

El odontólogo debe controlar la calidad y la cantidad de luz ambiental. Según Miller la luz artificial controlada a una temperatura Kelvin de alrededor de 5.500 o $\mathrm{K}$ y una intensidad de 175-200 bujías ofrece la máxima fiabilidad $^{15}$. Es esencial el control de estas dos dimensiones de la luz para conseguir una estandarización ya que la luz natural varía constantemente. Se puede utilizar la luz natural indirecta de buena calidad para aumentar la luz artificial.

Aunque para la objetividad y precisión, necesitemos un sistema instrumental colorimétrico para su valoración. Johnson, Seghi y cols, de-mostraron que era mejor el uso de un sistema colorimétrico, que el uso de un sistema visual ${ }^{16}$, ya que era más preciso y más fácil de reconocer las diferencias de color si usábamos un instrumento colorimétrico.

Dónde hay discrepancias entre autores, es a la hora de escoger el instrumento para reconocer las diferencias de color. Seghi y cols evaluaron tres maneras diferentes de captar instrumentalmente el color, usando dos colorímetros diferentes y un espectrofotómetro. Como resultado de dicho estudio surgió el concepto de que cualquiera de ellos es lo suficientemente válido y preciso para evaluar el color de la cerámica dental ${ }^{17 *}$.

Errores en la inadecuada reduc- ción del diente y la posterior construcción de la corona con insuficentes grosores producen fracturas post cementado que requieren de pulido intra oral de la cerámica, además existe la necesidad de realizar ajustes oclusales o de sobrecontorneado que requerirán un posterior pulido intraoral $^{17 *}$. Éste debastado y pulido influirá en la textura de la superficie, influyendo en la respuesta del periodonto a la restauración y en el color de la restauración, tal como veremos en la discusión de los resultados, siendo un factor importante si es una restauración en el sector anterior.

En la literatura hemos observado que el sistema de pulido más utilizado en los diferentes estudios es el sistema de pulido de la casa Shofu $\AA^{17 *, 18,19 *}$ Es por ello que hemos escogido este sistema de pulido, ya que nos permitirá comparar resultados con los estudios publicados.

Este experimento fue estructurado para eliminar todas las variables posibles de forma que se pudiese realizar una comparación objetiva, por ello todas las fases fueron evaluadas y realizadas por el mismo operador, ya que según O’Brien solo el cambio de operador al realizar una misma muestra produce una diferencia de color que el estimó en 3,5 unidades CIE-Lab ${ }^{13}$.

La discusión de los resultados es tarea ardua y difícil debido a que todos los estudios realizados en la literatura tienen parámetros diferentes, ya sea el grosor de la dentina, ya sean las unidades utilizadas, o los instrumentos de medición, además de la dificultad propia de analizar e imbrincar los resultados de los experimentos que hemos realizado.

El reglaseado de las restauraciones metalo-cerámicas debido a las fracturas postcementado de la cerámica, o bien la necesidad de realizar ajustes oclusales posteriores al cementado nos obligaran a determinar el tratamiento adecuado de la superficie cerámica.

Los estudios publicados en la literatura sobre pulido cerámico no son muchos, además de la existencia de mucha disparidad en la metodología utilizada. Algunos estudios solo se basan en microscopia electrónica ${ }^{5^{*}, 19^{*}}$.

La disparidad no solo está presente en la manera de medir las muestras, sino en las muestras en sí. De todos los experimentos encontrados en la literatura no hay ninguno comparable a otro debido a que se usan cerámicas diferentes, grosores diferentes, aleaciones diferentes, operadores diferentes y como hemos observado todas estas variables modifican la variable de color ${ }^{5 *, 17^{*}, 19^{*}}$.

La necesidad de reglasear toda superficie cerámica que ha estado manipulada en su capa externa con instrumentos abrasivos, ha estado en entredicho por diversos autores. Sulik después de un estudio de los acabados cerámicos, afirma que, en estudios basados en microscopia electrónica, las superficies pulidas dan resultados similares a las superficies glaseadas $^{5 *}$. Esta afirmación se contradice en los resultados de nuestro estudio en que si encontramos diferencias, ya sean colorimétricas como de superficie. Observando la imagen del microscopio electrónico de barrido vemos que sí existen diferencias de rugosidad de la superficie cerámica (figs.14 y 15).

Cabe destacar que las superficies glaseadas son mas abrasivas que las superficies que han sufrido un proce- 


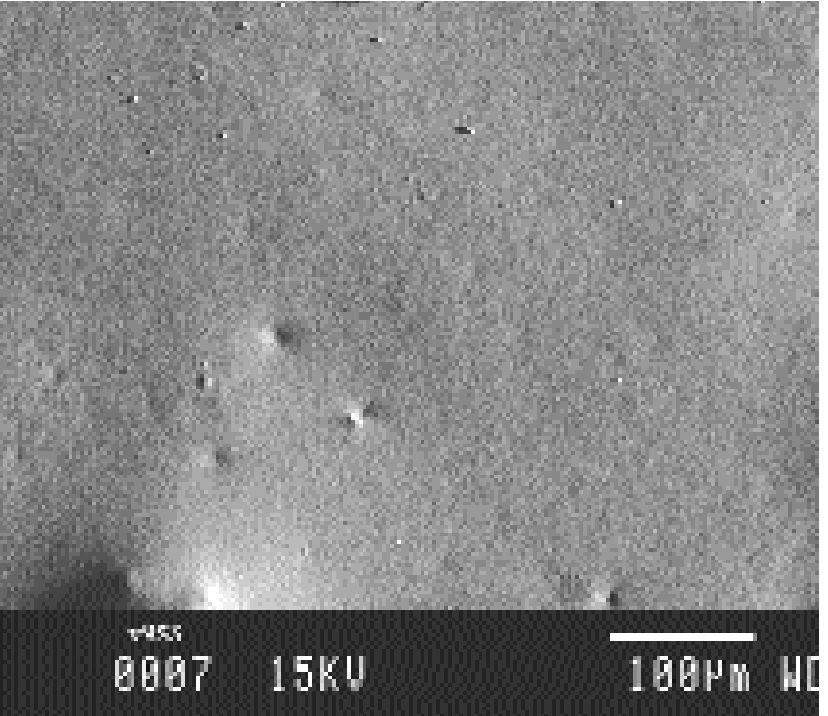

Figura 14: Cerámica glaseada vista con microscopia de barrido.

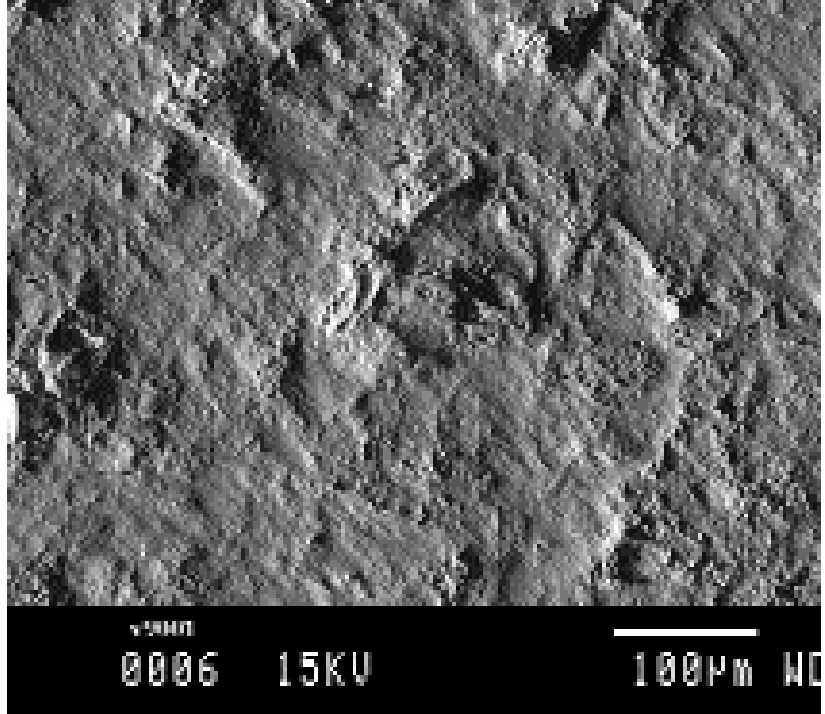

Figura 15:Aspecto de la cerámica pulida. so de pulido $^{20}$, produciendo pues un desgaste de los dientes antagonistas a la restauración más severo. Es por ello que algunos investigadores han recomendado eliminar el glaseado para disminuir la dureza e incluso mejorar la estética de la restauración ya que permite darle textura ${ }^{17^{*}, 21}$.

Kausner, en 1982, intentó definir y comparar cualitativamente el autoglaseado y el pulido de las superficies de la porcelana. Probó cuatro secuencias de pulido no encontrando diferencias significativas entre ellas; no obstante las diferencias significativas se encontraron entre las abrasiones comparadas previamente a la secuencia de pulido: las superficies pulidas con el kit de pulido Shofu son los que crean una superficie menos rugo$\mathrm{sa}^{22,23}$. Asi mismo, Golstein estudió varios métodos de pulido, obteniendo el kit de Shofu como clínicamente acceptable, además de verificar experimentalmente que las variables de la velocidad y presencia o no de agua no implican mejor eficacia de pulido $^{23}$.
Jagger mostró mediante un estudio estéreo microscópico como el glaseado dental se retiraba en dos días de abrasión intra-oral, dejando al descubierto las irregularidades de la porcelana subglaseada, actuando de una forma más abrasiva ${ }^{17 *}$. Palmer, en 1991, demostró como las restauraciones cerámicas maquilladas abrasionan el esmalte natural con más facilidad que la porcelana sin maquillaje, recomendando una cerámica vitrificada sin maquillaje $e^{23}$. De long describe que el desgaste es tres veces más en las restauraciones maquilladas que los no maquillados ya que aumenta la porosidad de la superficie ${ }^{24}$.

No obstante es patente en la literatura que la respuesta del tejido conectivo a la cerámica no glaseada es desfavorable, es por ello que quizás el pulido cerámico podría ser aceptado a nivel oclusal, pero no a nivel gingival ${ }^{18}$.

Después de analizar las diferencias colorimétricas pre y post pulido queda de manifiesto, en los resultados, que al igual que en los experimentos primeros las diferencias siempre son mas notorias en luz tipo tungsteno.

Otro de los resultados a valorar es que al pulir externamente la superficie cerámica la aleación que más cantidad color pierde es la aleación de alto contenido en oro. La interpretación clínica podría ser que la aleación que menos oxidación tiene es la que mas se ve influenciada por el pulido de la superficie cerámica, mientras que las que tienen más oxidación son las que menos varia el color.

Parece que la aleación en la que el color es mas estable después de pulir la superficie seria el «WIRON». Las dos aleaciones de cromo níquel siguen evoluciones diferentes que se supone se debe a la composición química.

La cerámica que más se ve influida por el proceso de pulido es la Vita VMK. Parece pues difícil dilucidar la imbrincación de todas las variables y su efecto final, ya que las combinaciones de todas ellas son múltiples, y todas influyen.

De cualquier manera, dada la gran cantidad de factores que intervienen en la formación de la imagen que 
tenemos del diente, consideramos que tendrán que desarrollarse muchos más estudios que permitan una mejor comprensión y manejo del color en la profesión dental.

\section{Conclusiones}

Después de haber planteado las hipótesis de trabajo, según el material y método expuestos, se pueden extraer las siguientes conclusiones:

1. El procedimiento usado es válido y apropiado para comparar diferencias de color.

2. Las diferencias de color varían según la luz usada y son mayores en una luz tipo tungsteno ( Luz A) que en una luz tipo día (Luz C).

3. Las diferentes aleaciones cromo níquel siguen comportamientos colorimétricos diferentes, su color varía de forma diferente después del pulido.

4. Existen diferencias de color entre aleaciones, cerámicas y tonos antes y después de un pulido de una superfície cerámica glaseada; siendo la aleación Cosmor la que más variación de color tiene y la aleación Wirón la que menos variación tiene. La cerámica que más ve influido su color por el pulido es la Vita VMK

A pesar de las conclusiones descritas en nuestro trabajo, consideramos que son necesarios estudios en mayor profundidad que permitan obtener un mayor conocimiento sobre la percepción visual de las diferencias de color originadas

\section{Bibliografía recomendada}

Para profundizar en la lectura de este tema, el/los autor/es considera/an interesantes los artículos que aparecen señalados del siguiente modo: *de interés **de especial interés.

1. Rosentiel SF, Land MF, Fujimoto J. Ciencia del color y selección del color en Prótesis Fija. Procedimientos clínicos y de laboratorio. Barcelona: ed Salvat, 1990:399-406.

2. Winter R., Cornell D. IPS d,Sign: la filosofia y manejo de una cerámica vítrea para metal. Signatura internacional; 2001;5:5-15.

3. Bonilla M. Revisión acerca de la abrasión y pulido intraoral de la cerámica dental. Tesina Universitat de Barcelona. 1995.

4. Barghi C, King CJ,Braughn RA. A Study of porcelain surfaces as utilized in fixed prosthodontics. J Prosthet Dent 1975;34:314-19.

5*. Sulik WD, Plekavich EJ. Surface finishing of dental porcelain. J Prosthet Dent. 1981;46(2): 217-21.

Realiza estudios sobre diversas supeficies cerámicas glaseadas y pulidas basadas en microscopia electrónica de barrido

6. Zalkind M, Lauer S, Stern N. Porcelain surface texture reduction and natural glazilnig. $\mathrm{J}$ prosthet Dent 1975;33:178-90.

7*. Bessing C, Wiktorsson A. Comparision of two different methods of polising porcelain. Scan J Dent Res 1983;91:482-7

Compara dos de los dos métodos más utlizados para el pulido cerámico post glaseado, compara su superficie final obtenido y concluye que no existen diferencias significativas entre ellos.

8. Monasky GE, Taylor DF. Studies of the wear of porcelain, enamel and gold. J Prosthet Dent 1971; 25(3):299-306.
9. Seghi RR, Johnson WM, O'Brien WJ. Spectrofotometric analysis of color between different porcelain systems. J Prosth Dent 1986;56:35-40.

10. Jacobs SH, Goodacre CJ, Moore BK, Dykema RW. Effect of porcelain thickness and type of metal-ceramic alloy on color. J Prosth Dent 1987;57:138-44.

11. Jagger DC, Raimondo RL, Richardson JT. Polished versus autoglazed dental porcelain. J Prosthet Dent 1990;64:553-7.

12. Stone MC. Esthetics and Color. JADA 1988; 116:613-4.

13. Egger B. El Status quo de la estética. Un análisis de los métodos y materiales para la reproducción del color. Quintessenz Técnica 1997;8:168-83.

14. Sproull RC. Color matching in dentistry, Part II. J Prosthet Dent 1973;23:556-65.

15. Miller LL. Programa de desarrollo en Odontología estética. J Esthet Dent 1994;6-16.

16. Johnson WM, Kao EC. Assessment of appearance match by visual observation and clinical colorimetry. J Dent Res 1989;68:819-22.

17*. Jagger DC, Harrison A. An in vitro investigation into the wear effects of unglazed, glazed, and polished porcelain on human enamel. J Prosthet Dent 1994;72:320-3

Compara el efecto de desgaste que se produce sobre el diente antagonista dependiendo de si el desgaste se produce sobre una superficie cerámica glaseada, superficie pulida o superficie no glaseada. Después de analizar los resultados obtenidos tras el desgaste e un simulador de ciclos masticatorios, se observa que el que menos desgaste produce al diente antagonista es la superficie pulida. Además se observa que la superficie glaseada desaparece en tan solo dos horas.

18. Goldstein GR, Barnahard BR, Penugonda B. Profilometer, SEM and visual assessment of porcelain polishing methods. $J$ Prosthet Dent 1991;65:627-34

19*. Klausner LH, Charbeneau GT. Polishing versus autoglaced porcelain surfaces. J Prosthet Dent 1982;47:157-62.

Evalúa diferentes secuencias de pulido y diferentes sistemas de pulido y no encuentra diferencias significativas en el resultado obtenido.

20. Jacobi R, Shillingburg HT, Duncanson MG. A comparison of the abrasiveness of six ceramic surfaces and gold. J Prosthet Dent 1991; 66:303-9.

21. Brewer JD, Garlapo DA, Chipps EA, Tedesco LA. Clinical discrimination between autoglazed and polished porcelain surfaces. J Prosthet Dent 1990;64:631-5.

22. Hulterström AK, Gergman M. Polishing systems for dental ceramics. Acta Odont Scand 1993;51:229-34

23. Palmer DS. Wear of human enamel against a comercial castable ceramic restorative material. J Prosthet dent 1991;65:192.

24. De Long R and cols. The wear of enamel opposing shaded ceramic restorative materials: An in vivo study. J Prosthet Dent 1992; 68: 42-8. 